\title{
Survival Analysis of Patients with End Stage Renal Disease the Case of Adama Hospital, Ethiopia
}

\author{
Mekiya Hussein ${ }^{1}$, Geremew Muleta ${ }^{2}$, Dinberu Seyoum ${ }^{2}$, Demeke Kifle ${ }^{2}$, Dechasa Bedada ${ }^{2}$ \\ ${ }^{1}$ Department of Statistics, Haramaya University, Haramaya, Ethiopia \\ ${ }^{2}$ Department of Statistics, Jimma University, Jimma, Ethiopia
}

Email address:

sofiyahusen@gmail.com (M. Hussein),yohager@gmail.com (G. Muleta), dinberu.seyoum@gmail.com (D. Seyoum), demeks10@gmail.com (D. Kifle), decheebe@gmail.com (D. Bedada)

\section{To cite this article:}

Mekiya Hussein, Geremew Muleta, Dinberu Seyoum, Demeke Kifle, Dechasa Bedada. Survival Analysis of Patients with End Stage Renal Disease the Case of Adama Hospital, Ethiopia. Clinical Medicine Research. Vol. 6, No. 6, 2017, pp. 201-208.

doi: $10.11648 /$ j.cmr.20170606.15

Received: October 18, 2017; Accepted: November 16, 2017; Published: December 15, 2017

\begin{abstract}
Background: Chronic kidney disease (CKD) with diagonesised end-stage renal disease (ESRD) is common public health problems worldwide. This study was aimed to investigate socio-economics and clinical characteristics determinants among end-stage renal disease (ESRD) population. Method: This study is a retrospective cohort design which was conducted during May 2012 to April 2016 and included 500 ESRD patients at Adama Hospital Medical College. Retrospectives data were gathered by reviewing patients' medical and surgical wards history. The Cox PH regression and parametric survival (Weibull, Log-logistic and log normal) models were molded and compared for examining survival analysis of ESRD patient using $\mathrm{R}$ statistical package software. Results: The study participants are 500 ESRD patients, $72.40 \%$ were alive at the end of this study, while $27.40 \%$ were died. The survival time of ESRD Majority of patients (66.20\%) were female. Log-normal model had fitted the ESRD data set best relatively among possible candidate models. The age at the time of admission to ESRD (HR $=0.94$, p-value < $0.05)$, female ( $H R=0.54, p$-value $<0.05)$ and family history $(H R=0.45$, $p$-value $<0.05)$ had significantly shorter survival time of ESRD patients to mortality. Conclusion: parametric survival model with baseline hazard lognormal distribution was found appropriate to our dataset. This study identified that having ESRD with complications increases the probability of death. The family history of experiencing ESRD is a driver for being ESRD patient. Female patients had greater risk of death than males in this study. Age specific follow-up is necessary to reduce the mortality related to ESRD.
\end{abstract}

Keywords: Chronic Kidney Disease, Risk Factors, Parametric Models, Ethiopia

\section{Background}

Chronic kidney disease (CKD) is a world-wide public health problem and it is associated with adverse outcomes of kidney failure, cardiovascular disease and premature death [1]. CKD is a condition in which your kidneys are damaged and cannot filter blood as well as healthy kidneys. During kidney failures, for a while, healthy nephrons can take on the extra work. But if the damage continues, more and more nephrons will be shut down. After a certain point, the nephrons that are left cannot filter your blood well enough to keep you healthy [2]. The CKD stages were categorized based on the classification system established by the National Kidney Foundation Kidney Disease Outcomes Quality Initiative classification. For the purposes of this study, CKD was defined as Kidney Disease Outcomes Quality Initiative CKD stages 1-5 [4, 5].

End-stage renal disease (ESRD) is the last stage of chronic kidney disease. This is when your kidneys can no longer support your body's needs. A person with ESRD needs treatment to replace the work of the failed kidneys [6]. According to the latest WHO data published in April 2011, kidney disease deaths in Ethiopia reached 12,038 or $1.47 \%$ of total deaths. The World Health Organization (WHO) reports also that for the first time in history deaths from chronic diseases will soon exceed deaths from communicable diseases, maternal and perinatal conditions, and nutritional deficiencies combined in low-income countries [1]. Kidney disease imposes disproportionate, incalculable human suffering and a catastrophic economic burden on the African continent in several respects: less than $2 \%$ of the 
patients with ESRD have access to RRT making ESRD a death sentence for most patients.

The ESRD rate is increasing at $6 \%$ to $8 \%$ per year in the African continent. Africa is experiencing an accelerated incidence of hypertension (60 million people) and type 2 diabetes mellitus ( $>12$ million people), which are the underlying causes in $>15 \%$ of CKD cases; and finally, at current estimates, none of the 54 countries in Sub-Saharan Africa (SSA) will be able to afford the cost of medical care associated with pre-dialysis CKD for their populations (estimated to be $\$ 2500$ to $\$ 20,000$ per patient annually) [5, $11,12]$. Globally, more than 100 countries (with combined population $>1$ billion) have no provisions for chronic maintenance dialysis or kidney transplantation and thus, more than 1 million people die annually from ESRD [6-8]. There is no cure for CKD, although treatment can slow or halt the progression of the disease and can prevent other serious conditions developing. [9, 13, 14]. This study aimed to determine what variables affect the probability of survival of Chronic Kidney Disease patients diagnosed with ESRD and to assess the effectiveness of the developed survival model to provide reasonably accurate.

The statistical analysis of lifetime data (time-to-event) plays an important role in medicine because it explore progression of event dynamically over time. There are two points related to this development of survival data. First, survival times are usually a mixture of discrete and continuous data that lend themselves to a different type of analysis. The Kaplan-Meier estimator of the survival function is a major and explanatory step in the development of suitable models for such kind of data. Second, most evaluations are made conditionally on what is known at the time of the analysis, and changes over time $[16,17]$.

The Cox proportional hazards $(\mathrm{PH})$ model is now the most widely used for the analysis of survival data in the presence of covariates or prognostic factors. One of the reasons this model is so popular is because of the ease with which technical difficulties such as censoring and truncation are handled. This is due to the appealing interpretation of the hazard function as a risk that changes over time [17]. The researcher use parametric survival models when the assumptions of Cox- $\mathrm{PH}$ should not fulfilled. Parametric survival models are statistically more powerful than nonparametric or semi-parametric models [19]. A survival analysis enables us to know the distribution of failure time which often described by using Weibull, Loglogistic and log normal statistical distributions. This study is pioneer which identified factors associated to CKD patients mortality, using retrospective study in Adama Hospital Medical College, Ethiopia.

\section{Methods and Materials}

\subsection{Source of Data and Study Design}

The data used for this study were obtained from Adama
Hospital Medical College, Adama town, Oromia regional state, Middle East Ethiopia. The survival data were extracted from the patient's chart which contains epidemiological, laboratory and clinical information of all CKD patients. Retrospectives data were gathered for patients in the medical and surgical wards. Data were collected on all patients from their medical records with laboratory result. The clinicians follow result from May 2012 to April 2016 in AHMC.

The response /dependent variable in this study is the survival time (time to death) measured in Days from the date of the treatment start until the date of the patient's death or censor (Patients who lost to follow; transferred to another Hospital for referral). The status of the patients is 1 if the person died and 0 if the person is censored over the given time. Some predictors are considered in this study to investigate the determinant factors for the survival time of CKD patients and are given below:- Sex ( $0=$ Male, $1=$ Female), Age (Years), Residence $(0=$ Rural, $1=$ Urban), Diabetes mellitus $0=$ No, $1=$ Type I, $2=$ Type II), Hipertensión $(0=$ No, $1=$ Yes $)$, Vascular access $(1=$ Fistula, 2= Catheter, 3=Graft), Family history $(0=$ Absent, $1=$ Present $)$, Obesity $(0=$ Absent, $1=$ Present $)$

\subsection{Non-Parametric, Semi-Parametric and Parametric Model}

The Kaplan-Meier (KM) method is a nonparametric survival analysis that is used to estimate the two survival probabilities curves dealing with differing survival times especially when not all of the subjects continue in the study. KM estimator incorporates information from all of the observations available, both censored and uncensored, by considering any point in time as a series of steps defined by the observed survival and censored times. When there is no censoring, the estimator is simply the sample proportion of observations with event times greater than $\mathrm{t}$. The technique becomes a little more complicated but still manageable when censored times are included. Let ordered survival times are given by $0 \leq t_{1} \leq t_{2}$ $\leq \mathrm{t}_{\mathrm{j}} \leq \infty$, then:

$$
\hat{S}(t)=\left\{\begin{array}{c}
1, \text { ift }<t_{1} \\
\prod_{j: t_{j \leq t}}\left[1-\frac{d_{j}}{r_{j}}\right]
\end{array}, \text { if } \mathrm{t} \geq \mathrm{t}_{1}\right.
$$

Where; $d_{j}$ is the observed number of events at time $t_{j}$ and $r_{j}$ is the number of individuals at risk at time $\mathrm{t}_{\mathrm{j}}$.

The Cox proportional hazards $(\mathrm{PH})$ regression model (introduced in a seminal paper by Cox, [20]; a broadly applicable and the most widely used method of survival analysis. Survival models are used to quantify the effect of one or more explanatory variables on failure time. This involves specification of a linear like model for the log hazard. A parametric model based on the exponential distribution may be parameterized as follows:

$$
h_{i}(t \mid x)=\exp \left(\alpha+\beta_{1} x_{i 1}+\beta_{21} x_{i 2}+\cdots+\beta_{k} x_{i k}\right)=\exp (\alpha) \exp \left(\beta^{\prime} X\right)
$$


In this case the constant $\alpha$ represents the log-baseline hazard since $\log h_{i}(t)=\alpha$ when the entries are zero. The Cox PH model is a semi-parametric model where the baseline hazard $\alpha(t)$ is allowed to vary with time.

$$
h_{i}(t \mid x)=h_{o}(t) \exp \left(X_{i}^{T} \beta\right)
$$

Where, $h_{o}(t)$ is the baseline hazard function; $\mathbf{X}_{\mathrm{i}}$ is a vector of covariates and $\beta$ is a vector of parameters for fixed effects.

The methods for verifying that a model satisfies the assumption of proportional hazard were graphical method.

However, there are two types of graphical techniques to check the proportional hazard assumptions. The most common technique is by comparing the estimated $\ln (-\ln S(t))$. If the two survival curves do not intersect and are parallel, it evidently provides evidence against the assumptions. Another graphical approach is by comparing observed with predicted survival curves. If the two survival curves are close, then the proportional hazard assumption is plausible

\subsection{Parametric Regression Models}

In the previous topics it was focused entirely on the use of semi-parametric model and proportional hazards Cox regression model, but a parametric survival model assumes that the survival time follows a known distribution. Many models using different distribution have been developed. The commonly applied models are log normal, weibull and log-logistic models.

Log Normal Regression Model

Skew distributions, low values mean high variance and non-negative values like species variety, distribution of minerals in earth's crust and generally based on log normal distribution are commonly used for responding to stimulant biologic substances, most types of survival data, time distribution of hardware repair, financial researchers and studying the load price. As the application of lognormal distribution was tested, there were some examples of geology, metallurgy, health, environment, ecology, linguistics, social sciences and economics [21].

\section{Results of the Study}

In this study, the totals of 500 ESRD patients were considered. Among those patients, $72.40 \%$ of them had been alive until the end of the study period, while $27.60 \%$ were died. Out of those male individuals in this study 169 (33.80\%), 32 (18.93\%) were died, and $106(32.02 \%)$ died individuals reported from $331(66.20 \%)$ female individuals. It also shows that there are more female ESRD patients than male patients.

The time-to-death of the ESRD patient's with different levels of the covariate were explored using Nonparametric survival analysis. This explanatory result gives information on the shape of the survival and hazard functions of ESRD data set. Survival time distributions for time-to-death was estimated for each covariates group using the K-M method and in order to compare the survival curves of two or more groups, log-rank test has been in use.

Table 1. Mean survival time, 95\% CI and long rank test of the ESRD from AMHC, 2016.

\begin{tabular}{|c|c|c|c|c|c|}
\hline \multirow{2}{*}{ Variable } & \multirow{2}{*}{ Categories } & \multirow{2}{*}{ Mean $[95 \% \mathrm{CI}]$} & \multicolumn{3}{|c|}{ Log rank test } \\
\hline & & & Chi. sq & Df. & P-value \\
\hline Age & & $53.34[51.9,54.7]$ & 149 & 69 & 0.000 \\
\hline \multirow{2}{*}{ Sex } & Male & $1445.78[1336,1555]$ & & & \\
\hline & Female & $1223.73[1137,1310]$ & 8.2 & 1 & 0.004 \\
\hline \multirow{2}{*}{ Residence } & Rural & $1381.95[1275,1488]$ & & & \\
\hline & Urban & $1250.38[1161,1339]$ & 2.3 & 1 & 0.128 \\
\hline \multirow{3}{*}{ Hypertension disease } & No & $1782.31[1747,1816]$ & & & \\
\hline & Yes & $1167.00[1085,1248]$ & 45.1 & 1 & 0.000 \\
\hline & No & $1790.86[1773,1808]$ & & & \\
\hline \multirow[t]{2}{*}{ Diabetic mellitus } & type one & $1040.88[891,1190]$ & 129 & 2 & 0.000 \\
\hline & type two & $923.49[804,1042]$ & & & \\
\hline \multirow{3}{*}{ Family history } & Absent & $1452.62[1337,1567]$ & & & \\
\hline & Present & $1235.00[1150,1319]$ & 7.2 & 1 & 0.007 \\
\hline & fistula & $1249.45[1128,1370]$ & & & \\
\hline \multirow[t]{2}{*}{ Vascular access } & catheter & $1339.79[1207,1472]$ & 0.9 & 2 & 0.624 \\
\hline & graft & $1308.83[1198,1419]$ & & & \\
\hline \multirow{2}{*}{ Obesity } & Absent & $1788.17[1765,1811]$ & & & \\
\hline & Present & $1107.46[1021,1193]$ & 68 & 1 & 0.000 \\
\hline Total & Overall mean & $1295.59[1226,1365]$ & & & \\
\hline
\end{tabular}

The mean survival time of ESRD patients with $95 \%$ confidence interval and different background characteristics of patients are summarized in Table 1. Accordingly, the mean survival time of male patients' to death was 1445.78 days, which is greater than female patients mean survival time 40.7 months. On the other hand the mean survival time to death of ESRD patients lived in the rural 46 months, $(95 \%$ CI: $[1275,1488])$ greater than from those of lived in the 42 months, with its' (95\% CI: [1275, 1488]). The mean survival time to death of ESRD patients, those who had no hypertension disease (60 months) were greater than from those ESRD patients with hypertension disease (39 months) respectively. Regarding the mean survival time to death (41 months) of the patients who had family history of kidney disease [FH-KD] was less than those patients those had no family history of kidney disease [FH-KD]. 

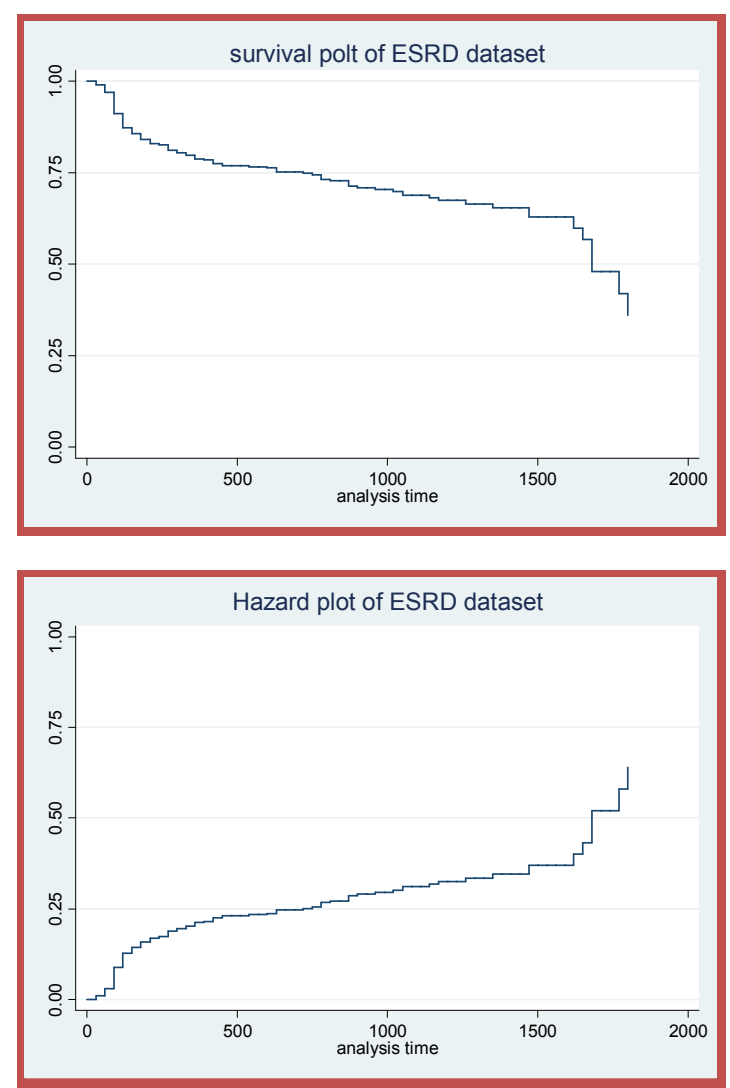

Figure 1. K-M plots of Survival and hazard functions of ESRD patients.
The mean survival time of the patients who had no diabetic mellitus were 60 months which is greater than those of categorized under type I diabetic (35 months) and type II diabetic (31 months) patients respectively. The mean survival time of patients' for vascular access user those treated through catheter (44 months) were greater than those treated through graft (43 months) and fistula (42 months) days respectively. Generally, the overall mean of survival time of ESRD patients was 43 months (Table 1), with 95\% confidence interval 40.8-45.5 months.

Plots of the KM curves to the survival and hazard experience of time- to- death is shown in Figure 1. The survival plot decline sharply at the beginning then go moves down slowly. This could show that there was higher number of deaths at the beginning of the start of the treatment. Thus, it implies that to investigate the he significance differences between the survival probabilities of patients by ESRD dataset.

Multivariate Analysis of Cox Ph Regression Model

Based on the $\mathrm{p}$ values of the output of individual covariate analysis at $10 \%$ significance level, some important predictor variables were not included in the model. Therefore, multiple covariate analysis has been done to check whether the excluded variables in univariate covariate analysis are significant. Table 2 shows the summary of multivariate analysis of Cox ph regression model.

Table 2. The Multivariate Cox PH Analysis to the ESRD dataset from the AHMC, 2016.

\begin{tabular}{|c|c|c|c|c|c|c|c|}
\hline Covariate & Category & $\operatorname{coef}(\beta)$ & HR & se (coef) & Wald & $\operatorname{Pr}(>|\mathbf{z}|)$ & [95\%Cl HR] \\
\hline Age & & 0.05 & 1.04 & 0.006 & 58.5 & 0.000 & {$\left[\begin{array}{ll}1.0 & 1.1]\end{array}\right]$} \\
\hline \multirow[t]{2}{*}{ Sex } & (0) male & Ref & & & & & \\
\hline & (1) female & 0.55 & 1.7 & 0.2 & 7.4 & 0.006 & {$\left[\begin{array}{ll}1.0 & 1.1\end{array}\right]$} \\
\hline \multirow[t]{2}{*}{ Residence } & (0) Rural & Ref & & & & & \\
\hline & (1) urban & 0.21 & 1.23 & 0.18 & 1.3 & 0.257 & {$[0.9,1.8]$} \\
\hline \multirow[t]{2}{*}{ Family history } & (0) Absent & Ref & & & & & \\
\hline & $\begin{array}{l}\text { (1) Present } \\
\text { (1) Fistula }\end{array}$ & $\begin{array}{l}0.63 \\
\text { Ref }\end{array}$ & 1.88 & 0.22 & 8.5 & 0.004 & [1.2 2.9] \\
\hline Vascular access & $\begin{array}{l}\text { (2) Catheter } \\
\text { (3) Graft }\end{array}$ & $\begin{array}{l}-0.18 \\
-0.21\end{array}$ & $\begin{array}{l}0.83 \\
0.81\end{array}$ & $\begin{array}{l}0.22 \\
0.2\end{array}$ & $\begin{array}{l}0.68 \\
11\end{array}$ & 0.409 & {$\left[\begin{array}{ll}0.5 & 1.3\end{array}\right]$} \\
\hline
\end{tabular}

Ref=reference, Coef $=$ coefficient, se $($ coef $)=$ standard error of coefficient; HR= hazard ratio; $p=$ value significant at $\leq 0.01$ level of significance.

Covariates which become insignificant in the multivariate analysis were removed from the model by using stepwise elimination technique. Therefore, residence and vascular access were not included as they are not significant predicators.

After adjusting other covariates, age, sex and family history were significantly associated to ESRD related mortality in multivariate Cox models. For one year increase in age, the ESRD patients mortality may increases by $4 \%$ $[\mathrm{HR}=1.04, \mathrm{P}=<0.01,95 \% \mathrm{CI}: 1.03,1.05]$, when the age of the patients increases by ten years (or he/she became 10 years older from where he/she has been) the hazard ratio is 1.65 $\left[H R=e^{(\beta a g e)}=e^{0.05 a g e * 10}=1.649\right.$ age $\left.\approx 1.65\right]$. This means the hazard of acquiring the ESRD mortality increases by $65 \%$. Thus, in this study, age was one of the most important contributors to patient's hazard in ESRD patients. The chance of acquiring mortality in ESRD was higher in female patients $70 \%$ than male patients. Family history of experiencing ESRD increased the mortality rate by $88 \%$ $[\mathrm{HR}=1.88,(90 \% \mathrm{CI} ;[1.2,2.9]), \mathrm{p}<0.01]$.

Table 3. The AIC and Log-Likelihood value for different parametric regression models.

\begin{tabular}{llll}
\hline Models type & Weibull & Log-logistic & Log normal \\
\hline AIC values & 721.4 & 710 & 703.9 \\
Log-Likelihood & -350.7 & -345 & -341.9 \\
\hline
\end{tabular}

AIC $=$ Akaike's information criteria.

The common applicable criterion to select the model is the Akaikie information criterion (AIC) statistic. Based on the finding (Table 3), the log normal regression model has the least AIC value which shows that the log normal regression model well fitted ESRD dataset. And, the Cox snell plot below in Figure 2 \& Figure 3 also indicates the log normal model fit the data better than the rest parametric models. 
Based on both criteria (AIC and Cox-Snell residual), log normal survival model had the best fit compared to other parametric models, such as Weibull and log-logistic. Based on the lognormal model, age of patients $(\mathrm{HR}=0.94$, $\mathrm{p}$-value $<$
$0.05)$, Female $(\mathrm{HR}=0.5$, $\mathrm{p}$-value $<0.05)$, family history $(\mathrm{HR}=0.5, \mathrm{p}$-value $<0.05)$, Residence $(\mathrm{HR}=0.7 \mathrm{p}$-value $>0.05)$ were significant covariates.

Table 4. The Log normal regression model of ESRD of AHMC, 2016.

\begin{tabular}{llllllll}
\hline Covariate & Category & $\widehat{\boldsymbol{\beta}}$ & Std. Err. & Wald & RR & P>|z| & [95\% CI RR] \\
\hline Age & Male & -0.06 & 0.01 & 53 & 0.94 & 0.000 & {$[0.930 .96]$} \\
Sex & $\begin{array}{l}\text { Ref } \\
\text { Female }\end{array}$ & -0.63 & 0.13 & 6.2 & 0.53 & 0.013 & {$[0.330 .88]$} \\
Family history & $\begin{array}{l}\text { Absent } \\
\text { Present }\end{array}$ & $\begin{array}{l}\text { Ref } \\
-0.80\end{array}$ & 0.12 & 9.1 & 0.45 & 0.003 & {$[0.270 .76]$} \\
\hline
\end{tabular}

$\beta=$ coefficient, se (coef) =standard error of coefficient; $R R=$ relative risk; $\mathrm{p}=$ value significantat $\leq 0.01$ level of significance.

Survival time of ESRD patients were significantly related with age, sex of patients, and family history of kidney disease. Using the adjusted multivariate model, the hazard ratio of a patient with the related risk of ESRD age of patients is 0.94 . The related risk of ESRD female patients is decreased by 0.54 times than ESRD male patients. The related risk of family history of kidney disease is decreased by 0.45 than no family history of kidney disease.

The Cox snell plot indicates the data was better fitted by $\log$ normal distribution since the 45 degree lines are more expressed by $\log$ normal distribution. Table 3 showed methods to select a model that fit the ESRD dataset based on the AIC value. The Cox snell plot method over time revealed that the hazard ratio is constant over time.
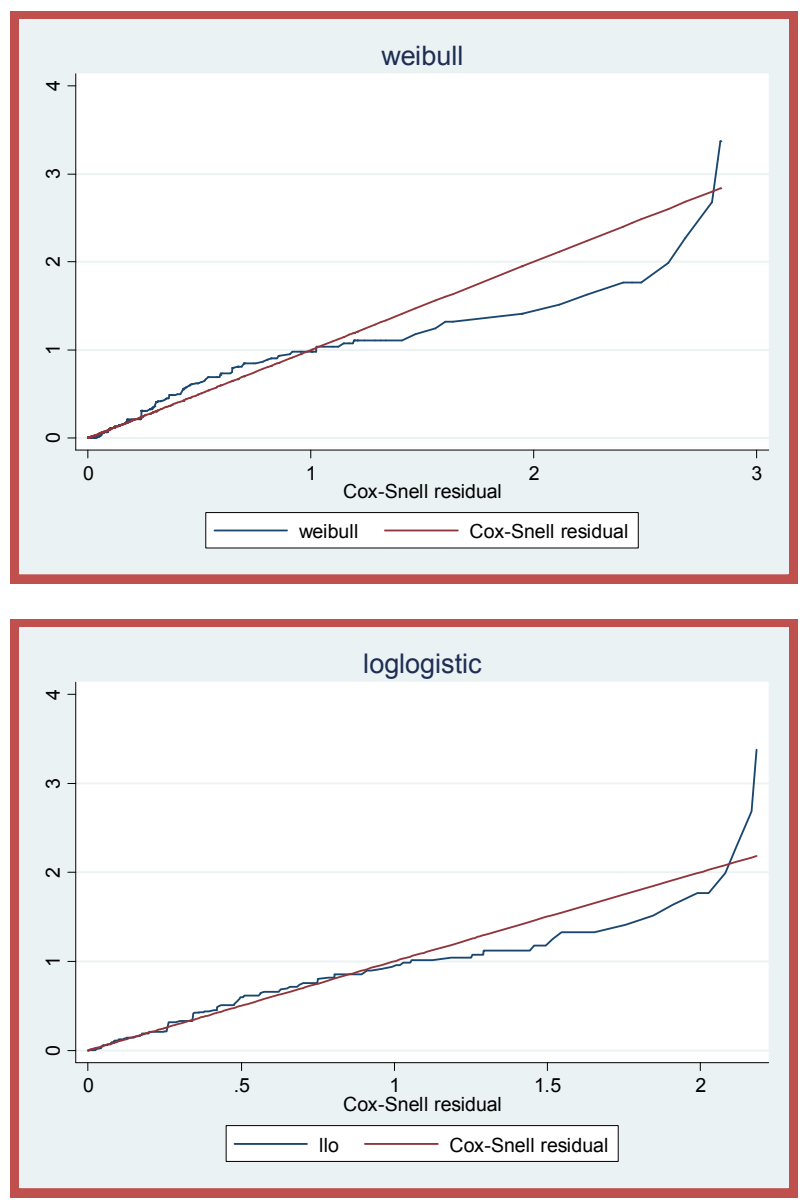

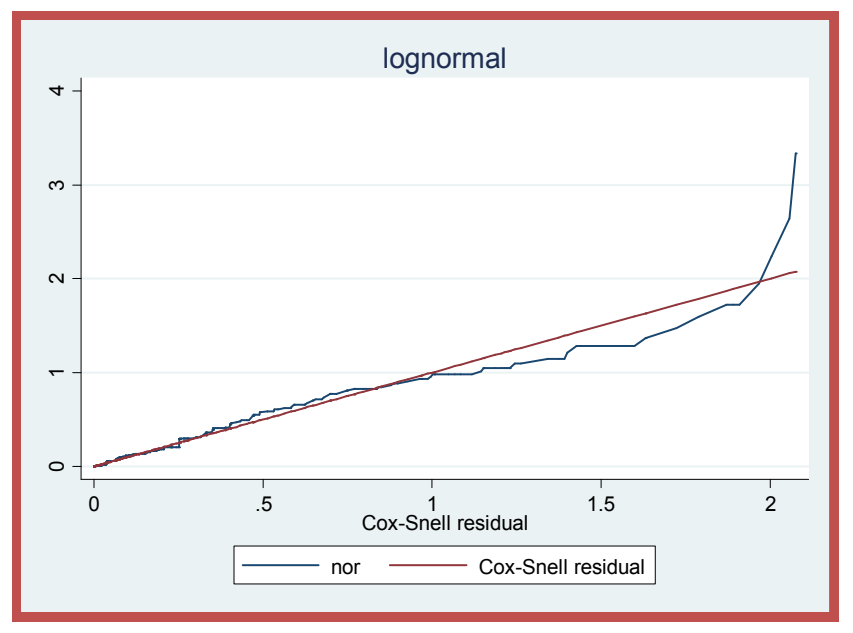

Figure 2. Cox-snell residual obtained using Weibull, Log-logistic, and Log normal model to the ESRD dataset.

As Figure 3 below, the deviance residual against the linear predication shows that the deviance residuals seem to be approximately symmetric distributed about zero and there exists no clearly outlying observation. This shows the adequacy of the fitted log normal model.

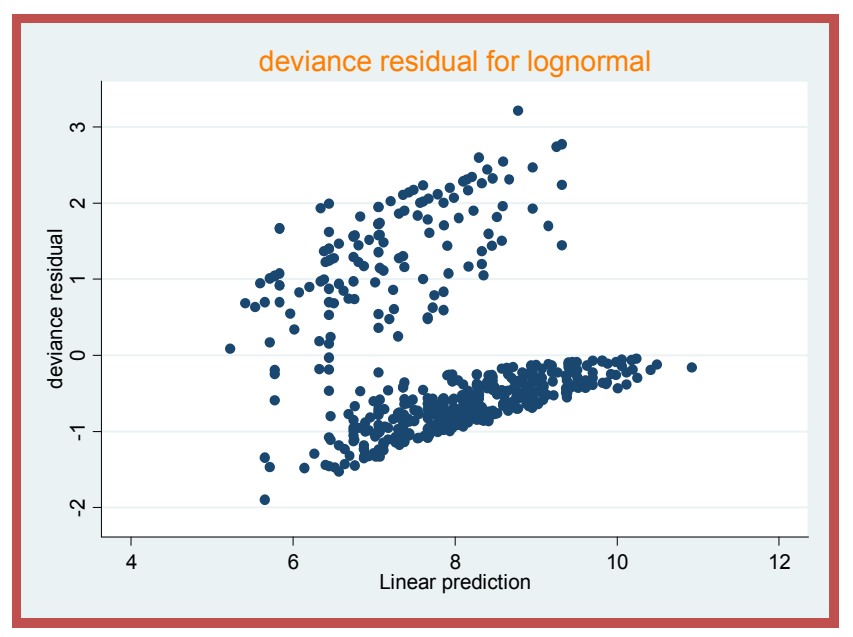



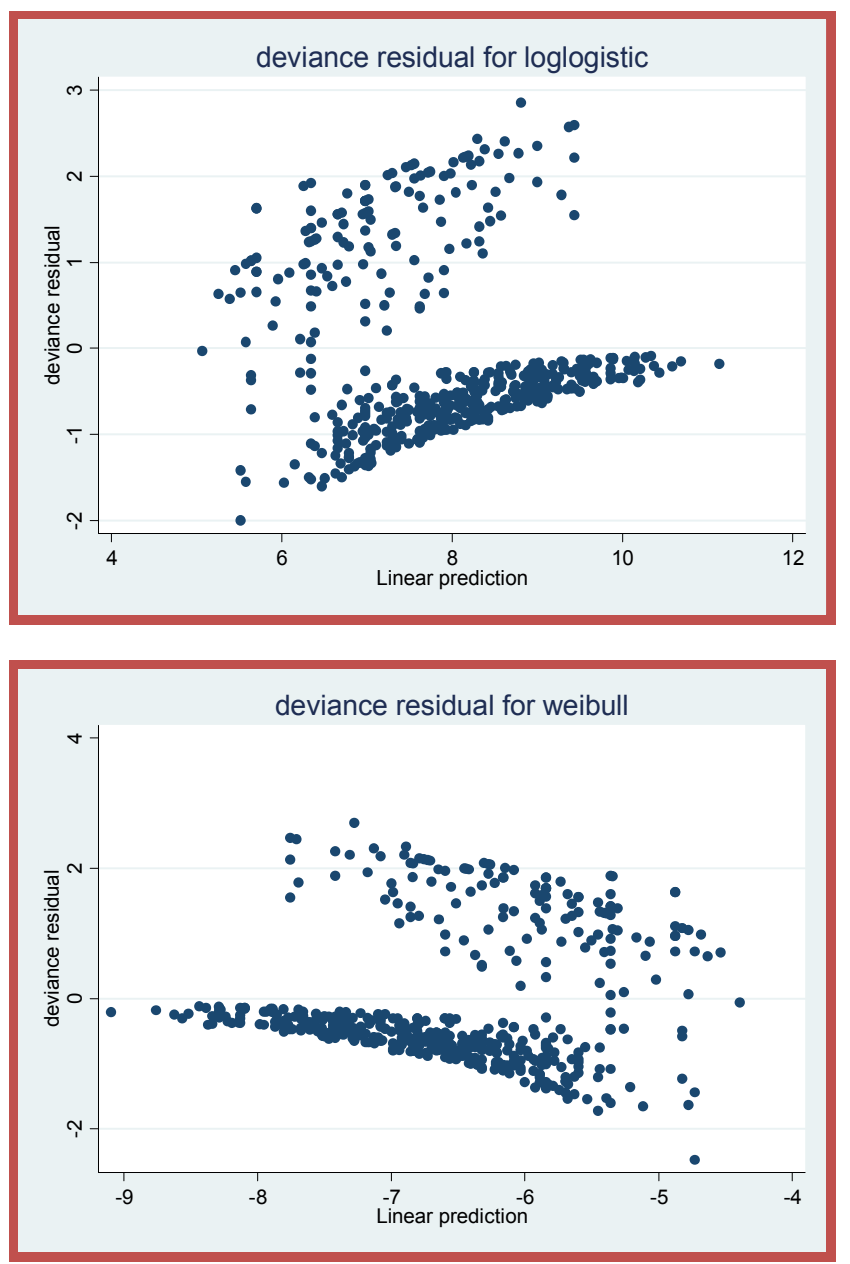

Figure 3. Deviance residual obtained by fitting Weibull, Log-logistic, and Log normal model for survival time-to ESRD data set.

\section{Discussion}

In this study to estimate and compare the Cox ph regression and parametric survival models by considering three baseline distributions: Weibull, log-logistic, and lognormal distributions. For this study the source of data were in a single center study with 500 common and occurrence end stage renal disease patients. It presented the results of 5 years patient survival and risk factors of mortality during a mean of follow-up of 43 months. Covariate which were included in the study were age, sex, residence, hypertension, diabetic mellitus, family history, vascular access and obesity of patients and the outcome variable of interest were the survival of time-to-death of the ESRD patients in days.

Most of the patients with ESRD at the hospital had prior diagnosis of diabetes mellitus and hypertension with complexity obesity. It is even more significant for developing countries which now face the double burden of infectious diseases and growing problems of non-communicable diseases such as obesity, diabetes and hypertension [22]. These factors in country study and abroad were found to be the most common cause of CKD [1, 23, 24] as there was no routine renal biopsy and other necessary investigations for diagnosis of renal diseases, the real causes of ESRD in this setting cannot be easily stated [23].

The Cox's proportional hazard model fitted using complete case analysis found five variables that can serve as predictive factors on the survival analysis of with ESRD. These are age, sex of the patients, residence, family history of kidney disease and vascular access. With regard to the parametric regression models also included in this study which do not assume constant baseline hazard except for exponential regression model. This is consistent with findings from other studies. [25-27]

The hazard of ESRD female patients was $70.00 \%$ greater than male patients. Sex differences have a significant association with ESRD in this study when renal function was assessed by the in Univariate and Multivariate Cox models. But, not with the Japanese's study in which the male gender was reported to be a non-modifiable risk factor for CKD [28]. Furthermore there was a strong association between female sex and CKD in the UK and Sweden studies [29]. The possible explanation for this might be due to the higher proportion of obese females than males.

The hazard of family history kidney disease was $88 \%$ greater than no family history $[\mathrm{HR}=1.88,90 \% \mathrm{CI}:(1.2,2.9)$, $\mathrm{p}<0.01]$, There was a significant association between ESRD and the presence of longer duration a family history of kidney disease in this study in log rank test and Cox ph model. This corresponds with the findings of several studies, which reported that the likelihood of developing reduced GFR was greater among patients with longer duration of diabetes and among those with or whose parents has kidney disease $[1,27,30]$.

In other way, various parametric models like Weibull, lognormal and log-logistic are widely used for analyzing the survival data. These models can interpret the survival time based on a specific distribution irrespective of proportional hazard hypothesis. This makes stronger the survival data analysis using parametric models. This means that, under special conditions, parametric models such as Weibull, loglogistic and lognormal may have more accurate results than Cox model [31]. Therefore: parametric, non-parametric or semi-parametric models. Moreover, it has more flexibility in adding covariates to the model. In this study, some variables that were significant through the log-rank test and had the proportional hazard assumption were introduced into the model.

By using AIC value, the models developed from Weibull, lognormal, log-logistic and Cox model were evaluated [32]. Results indicated the general preference of parametric models over Cox semi-parametric model. Among parametric models, than others did from log-logistic, lognormal and Weibull models with close values, the lognormal model had the lowest AIC quantity. Thus, lognormal model was the best fit model over the data of ESRD survival time in this research. This result is in line with the previous study in [31]. However, all survival data set should be warranted for possible survival time distribution assumptions before applying elected model. 


\section{Conclusions}

Parametric survival model with baseline hazard lognormal distribution was found appropriate to our dataset. This study identified that having ESRD with complications increases the probability of death. The family history of experiencing ESRD is a driver for being ESRD patient. Female patients had greater risk of death than males in this study. Age specific and gender-tailored follow-up is necessary to reduce the mortality related to ESRD.

\section{References}

[1] Temesgen F., Mehidi K, Tilahun Y. (2014). Prevalence of chronic kidney disease and associated risk factors among diabetic patients in southern Ethiopia, American Journal of Health Research, 2 (4): 216-221 (http://www.sciencepublishinggroup.com/j/ajhr).

[2] James MT, Quan H, Tonelli M, et al. (2009 Jul); CKD and risk of hospitalization and death with pneumonia. American Journal of Kidney Diseases. 54 (1): 24-32. PMID19447535.

[3] Atkins RC, Zimmet P. World Kidney Day 2010 Feb: Diabetic Kidney Disease-Act Now or Pay Later. Am J Kidney Dis. 55 (2): 205-8.

[4] Zilisteanu, D. S.(2013) Late nephrology referral and impact on morbidity and mortality of patients with chronic renal disease, $\mathrm{Ph}$. D. Thesis, Carol Davila University of Medicine and Pharmacy, Bucharest.

[5] National Kidney Foundation. (2002 Feb); K/DOQI clinical practice guidelines for chronic kidney disease: evaluation, classification, and stratification. Am J Kidney Dis. 39 (2 Suppl 1): S1-266. PMID 11904577.

[6] USRDS. United States Renal Data System 2008 Annual Data Report: Atlas of Chronic Kidney Disease and End-Stage Renal Disease in the United States. Bethesda, MD: National Institutes of Health, National Institute of Diabetes and Digestive and Kidney Diseases; www.usrds.org/adr_2008.htm. Accessed on Jan. 2010.

[7] Gilbertson DT, Liu J, Xue JL, et al. (2015); projecting the number of patients with end stage renal disease in the United States to the year. Journal of the American Society of Nephrology; 16 (12): 3736-41. PMID 16267160.

[8] USRDS. United States Renal Data System 2010 Annual Data Report: Atlas of Chronic Kidney Disease and End-Stage Renal Disease in the United States. Bethesda, MD: National Institutes of Health, National Institute of Diabetes and Digestive and Kidney Diseases.

[9] Zewdu W. (1994) Acute renal failure in Addis Ababa, Ethiopia: a prospective study of 136 patients. Ethiop Med J; 32 (2): 79-87.

[10] Wali RK. (2010) Aspirin and the prevention of cardiovascular disease in chronic kidney disease: time to move forward? J Am Coll Cardiol; 56: 966-8.

[11] Schieppati A, Remuzzi G. (2003) the future of renoprotection: frustration and promises. Kidney Int; 64: 1947-1955

[12] Zhang L, Wang F, Wang L, Wang W, Liu B, Liu J, Chen M,
He Q, Liao Y, Yu X, Chen N, Zhang JE, Hu Z, Liu F, Hong D, Ma L, Liu H, Zhou X, Chen J, Pan L, Chen W, Wang W, Li X, Wang H; (2012) Prevalence of chronic kidney disease in China: a cross-sectional survey. Lancet, 379: 815-822.

[13] Randeree IG, Czarnocki A, Moodley J, Seedat YK, Naiker IP. (1995) Acute renal failure in pregnancy in South Africa. Ren Fail; 17 (2): 147-153.

[14] Cheng, C. L., Kao, Y. H., Lin, S. J., Lee, C. H. \& Lai, M. L. (2011) Validation of the National Health Insurance Research Database with ischemic stroke cases in Taiwan. Pharmacoepidemiology and drug safety 20, 236-242.

[15] Naicker S. End-stage renal disease in sub-Saharan Africa. Ethn Dis 2009; 19 (S1): 13-15.

[16] Kaplan, E. L., and P. Meier. (1958). Nonparametric estimation from incomplete observations. Journal of the American Statistical Association 53: 457-481.

[17] Oakes D. (1977), the asymptotic information in censored survival data. Biometrika. 64, 441-8.

[18] Lawless, J. F., (1982). Statistical Methods and Model for Lifetime Data; Wiley, New York.

[19] Kleinbaum D, Klein M. Survival analysis: A self- learning text, New York, Springer-Verlag. 2005.

[20] Kalbfleisch, J. D., and Prentice, R. L. Marginal likelihoods based on Cox. s regression and life model. Biometrika 60 (1973), 267.278.

[21] US Renal Data System. USRDS 2002 Annual Data Report. Bethesda: National Institutes of Health, National Institute of Diabetes and Digestive and Kidney Diseases.

[22] McGilchrist, C. A. and Aisbett, C. W. (1991), Regression with frailty in survival analysis. Biometrics 47, 461-466.

[23] Fatiu A, Rashad S. (2008) CKD Prevention in Sub-Saharan Africa: A Call for Governmental, Nongovernmental, and Community Support. Am J. Kidney Dis. 51: 515-523. doi: 10.1053/j.ajkd.2007.12.006? [PubMed].

[24] Tamiru Sh., Esayas K., Belete H., Amare D. and Tewodros A., (2013) Survival patterns of patients on maintenance Hemodialysis for end stage renal disease in Ethiopia: summary of 91 cases, BMC Nephrology. 14: 127, PMCID: PMC3693969.

[25] IBRAHIM, J. G., CHEN, M.-H., and SINHA, D. Bayesian Survival Analysis. Springer-Verlag, New York, 2001.

[26] Go AS, Chertow GM, Fan D, et al. (2004 Sep 23) Chronic kidney disease and the risks of death, cardiovascular events, and hospitalization. New England Journal of Medicine; 351 (13): 1296-305. PMID 15385656.

[27] Laird, N., and Olivier, D. (2012) Covariance analysis of censored survival data using log-linear analysis.

[28] Ohta M, Babazono T, Uchigata Y, Iwamoto Y. (2010) Comparison of the prevalence of chronic kidney disease in Japanese patients with Type 1 and Type 2 diabetes. Diabet Med. 27 (9): 1017-23.

[29] Retnakaran R, Cull CA, Thorne KI, Adler AI, Holman RR. (2006) Risk Factors for Renal Dysfunction in Type 2 Diabetes U. K. Prospective Diabetes Study 74. Diabetes. 55 (6): 1832 9. 
[30] Klein, J. P., and Moeschberger, M. L. (1997) Survival Analysis: Techniques for Censored and Truncated Data. Springer, New York.

[31] Limpert, E., W. A. Stahel and M. Abbt, 2001. 24. Nardi, A. and M. Schemper, 2003. Log-normal distributions across the sciences: keys and clues. Biosciences, 51 (5): 341-52.

[32] Therneau T, Grambsch P. (2000) Modeling survival data: Extending the Cox Model, New York, Springer-Verlag. 